Article

\title{
Impact of Clostridioides difficile Therapy on Nosocomial Acquisition of Vancomycin-Resistant Enterococci
}

\author{
Carlos L. Correa-Martínez ${ }^{1,+}+\mathbb{D}$, Niklas C. J. Hagemeier ${ }^{1,+}{ }^{+}$, Neele J. Froböse ${ }^{2}$ and Stefanie Kampmeier ${ }^{1, *(\mathbb{D})}$ \\ 1 Institute of Hygiene, University Hospital Münster, Robert-Koch-Straße 41, 48149 Münster, Germany; \\ Carlos.Correa@ukmuenster.de (C.L.C.-M.); Niklascarljosef.Hagemeier@ukmuenster.de (N.C.J.H.) \\ 2 Institute of Medical Microbiology, University Hospital Münster, Domagkstraße 10, 48149 Münster, Germany; \\ Neelejudith.Froboese@ukmuenster.de \\ * Correspondence: Stefanie.Kampmeier@ukmuenster.de; Tel.: +49-251-83-52316 \\ + Equally contributing first authors.
}

Citation: Correa-Martínez, C.L.; Hagemeier, N.C.J.; Froböse, N.J.; Kampmeier, S. Impact of Clostridioides difficile Therapy on Nosocomial Acquisition of Vancomycin-Resistant Enterococci. Pharmaceuticals 2021, 14, 1066. https://doi.org/10.3390/ ph14111066

Academic Editor: Magdolna Csávás

Received: 27 September 2021

Accepted: 20 October 2021

Published: 21 October 2021

Publisher's Note: MDPI stays neutral with regard to jurisdictional claims in published maps and institutional affiliations.

Copyright: (c) 2021 by the authors. Licensee MDPI, Basel, Switzerland. This article is an open access article distributed under the terms and conditions of the Creative Commons Attribution (CC BY) license (https:// creativecommons.org/licenses/by/ $4.0 /)$.

\begin{abstract}
Vancomycin is frequently used for the treatment of $C$. difficile infections (CDI). There are concerns that this might increase the risk of selecting vancomycin resistant enterococci (VRE). Here, we evaluated whether there is an increased risk of VRE acquisition following vancomycin for CDI specific treatment. Patients with CDI, metronidazole, or oral vancomycin treatment and without preexisting VRE were monitored for VRE acquisition. VRE isolates from patients with acquired and preexisting colonization were collected and subjected to whole genome sequencing. In total, 281 patients (median age 56 years, $54 \%$ of the male sex) presented with toxin positive $C$. difficile. Of them, 170 patients met the inclusion criteria, comprising 37 patients treated with metronidazole and 133 treated with oral vancomycin. In total, 14 patients meeting the inclusion criteria acquired VRE (vancomycin: $n=11$; metronidazole: $n=3$ ). Statistical analysis revealed no significant differences between both VRE acquisition rates. Genetic comparison of detected VRE isolates resulted in eight clusters of closely related genotypes comprising acquired and preexisting strains. Our results suggest that vancomycin and metronidazole likewise increase the risk of VRE acquisition. Genetic comparison indicates that VRE acquisition is a result of both antibiotic selection and pathogen transmission.
\end{abstract}

Keywords: CDI; VRE; antimicrobial stewardship; whole genome sequencing

\section{Introduction}

In past decades, Clostridioides difficile infections (CDI) have evolved into one of the most common healthcare-associated infections worldwide. In Germany, where surveillance of these infections is mandatory, an incidence of 0.47 cases $/ 1000$ patient days could be observed in 2020 , of which $11 \%$ were classified as severe cases following the criteria of the German infection protection law [1,2].

According to common guidelines, therapeutic management of $C$. difficile infections depends on the severity of symptoms and the number of previous episodes. While formerly metronidazole has been considered the first-line agent treatment of non-severe CDI, since 2021, fidaxomicin and alternately vancomycin are assessed as superior for these indications in the current clinical practice guidelines from the Infectious Diseases Society of America and the Society for Healthcare Epidemiology of America [3]. However, metronidazole was preferred for mild or moderate infections previously but is now considered for these indications in the latest guideline release of the European Society of Clinical Microbiology and Infectious Diseases (ESCMID) [4]. Hence, in the University Hospital Münster (UHM), non-severe CDI infections are treated with metronidazole, while patients with severe CDI infections receive oral vancomycin.

While the choice of antibiotic agents for different clinical presentations is clearly supported by recent studies [5-7], its implications need to be further evaluated. Here, alongside economic health aspects, topics of infection prevention, such as the acquisition 
of multidrug resistant pathogens, need to be considered. Effects of antibiotics on nosocomial epidemiology of vancomycin-resistant enterococci (VRE) are thereby controversially discussed. There is evidence that the intravenous use of vancomycin is a major risk factor for VRE colonization [8,9] and has been associated with long-term VRE shedding [10]. On the other hand, in the absence of vancomycin therapy, VRE acquisition rates were shown to be ca. $1 \%$ at baseline but increased to over $6 \%$ after ertapenem application [11]. Additionally, oral vancomycin for $C$. difficile treatment did not result in enhanced VRE carriage rates compared to metronidazole treatment in a multicenter retrospective cohort study conducted in the USA [12].

VRE is an emerging pathogen posing a major threat to healthcare systems. Infections with VRE lead to a significantly higher mortality than infections with vancomycin susceptible enterococci [13,14]. Several risk factors for healthcare-associated acquisition of VRE are already characterized $[15,16]$. Alongside comorbidities, therapeutic interventions, and suboptimal implementation of hygiene measures, environmental persistence and subsequent nosocomial acquisition can be favored by pathogen-associated factors $[17,18]$. It is conceivable that VRE with specific genetic characteristics are preferably selected in CDI patients receiving antibiotic agents. To examine this hypothesis, we compared VRE colonization rates between previously VRE-negative patients receiving either metronidazole or oral vancomycin as a CDI-specific treatment. VRE isolates of patients with preexisting VRE colonization were compared with those of patients colonized after CDI-specific treatment.

\section{Results}

During 2018 and 2020, 281 patients (median age 56 years, 54\% of male sex) presented with toxin positive CDI, of which 31 were classified as severe cases and 175 as hospitalacquired cases due to the above-mentioned criteria. Of all CDI patients, 145 received oral vancomycin and 40 received metronidazole for CDI-specific treatment. In total, 111 patients did not fulfill the inclusion criteria and had to be excluded due to CDI treatments being different to metronidazole or oral vancomycin mono-treatment $(n=82)$ or preexisting VRE colonization $(n=29)$ (Figure 1).

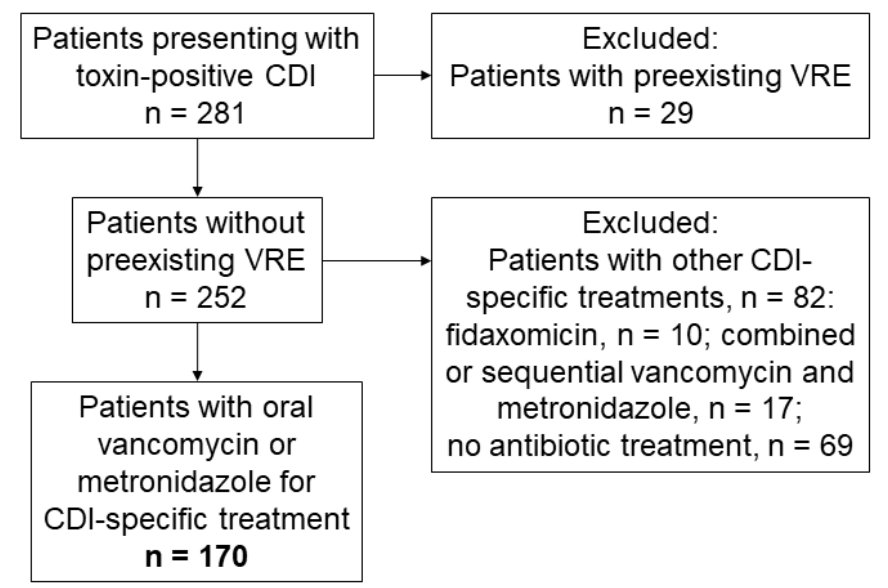

Figure 1. CDI patients meeting inclusion criteria.

\subsection{Characteristics of Included Patients and Onset of VRE}

In total, 170 patients (median age 53 years, $55 \%$ of male sex) met the inclusion criteria and were further observed. Clinical characteristics potentially favoring VRE can be gathered from Table 1. Of all included patients, 133 received oral vancomycin treatment $(4 \times 250 \mathrm{mg} /$ day $)$, while in 37 patients metronidazole $(3 \times 500 \mathrm{mg} /$ day $)$ was the preferred antibiotic agent. In total, 14 patients (metronidazole: 3; vancomycin: 11) acquired VRE after the first application of CDI-specific treatment. Statistical analysis did not result in significant differences between VRE acquisition rates of patients that received treatment with metronidazole and those treated with oral vancomycin $(p=0.98)$. VRE acquisition 
occurred approximately 21.5 days (metronidazole: 14 days; vancomycin: 25 days) after CDI-specific therapy.

Table 1. Comparison of demographic and clinical characteristics of patients meeting the inclusion criteria and treated with metronidazole or oral vancomycin $(n=170)$.

\begin{tabular}{ccc}
\hline Characteristic & \multicolumn{2}{c}{ Value (\%) } \\
\cline { 2 - 3 } & Metronidazole $(\boldsymbol{n}=\mathbf{3 7})$ & Vancomycin $(\boldsymbol{n}=\mathbf{1 3 3})$ \\
\hline Demographic data & 50 \\
Median age (years) & 58 & $72(54)$ \\
Male gender & $22(59)$ & $57(43)$ \\
Underlying diseases & & $57(43)$ \\
Haemato-oncological diseases & $22(59)$ & $12(9)$ \\
Immunosuppressive disease & $23(62)$ & $6(5)$ \\
Hepatic insufficiency & $5(14)$ & $30(23)$ \\
Liver Transplantation & $1(3)$ & $12(9)$ \\
Renal insufficiency & $6(16)$ & $36(27)$ \\
Long term dialysis & $1(3)$ & $79(59)$ \\
Treatment & $6(16)$ & \\
Systemic glucocorticoid treatment & $20(54)$ & 126 \\
Non-CDI-specific antibiotic & & 4 \\
treatment & 67 & \\
Contact to healthcare system & 2 & \\
Average lengths of stay (days) & & \\
Median number of stays & & \\
\hline
\end{tabular}

\subsection{VRE Genotypes and Genetic Distribution of Strains}

Of 45 VRE isolates, 43 (16 acquired and 27 preexisting) isolates were available for WGSbased typing. Out of these, $40(93 \%)$ harbored vanB and $3(7 \%)$ harbored vanA. Among all samples, prevalent MLST STs were ST117 (39 isolates, 91\%), ST262 (2 isolates, 5\%), ST192, and ST721 (1 isolate each, 2\%). CgMLST-based typing resulted in eight clusters of genetically closely-related genotypes comprising 2-8 genotypes (Figure 2, grey highlights), indicating possible intra-hospital VRE transmissions. However, there was no specific clustering of isolates associated with acquired or preexisting VRE (Figure 2).

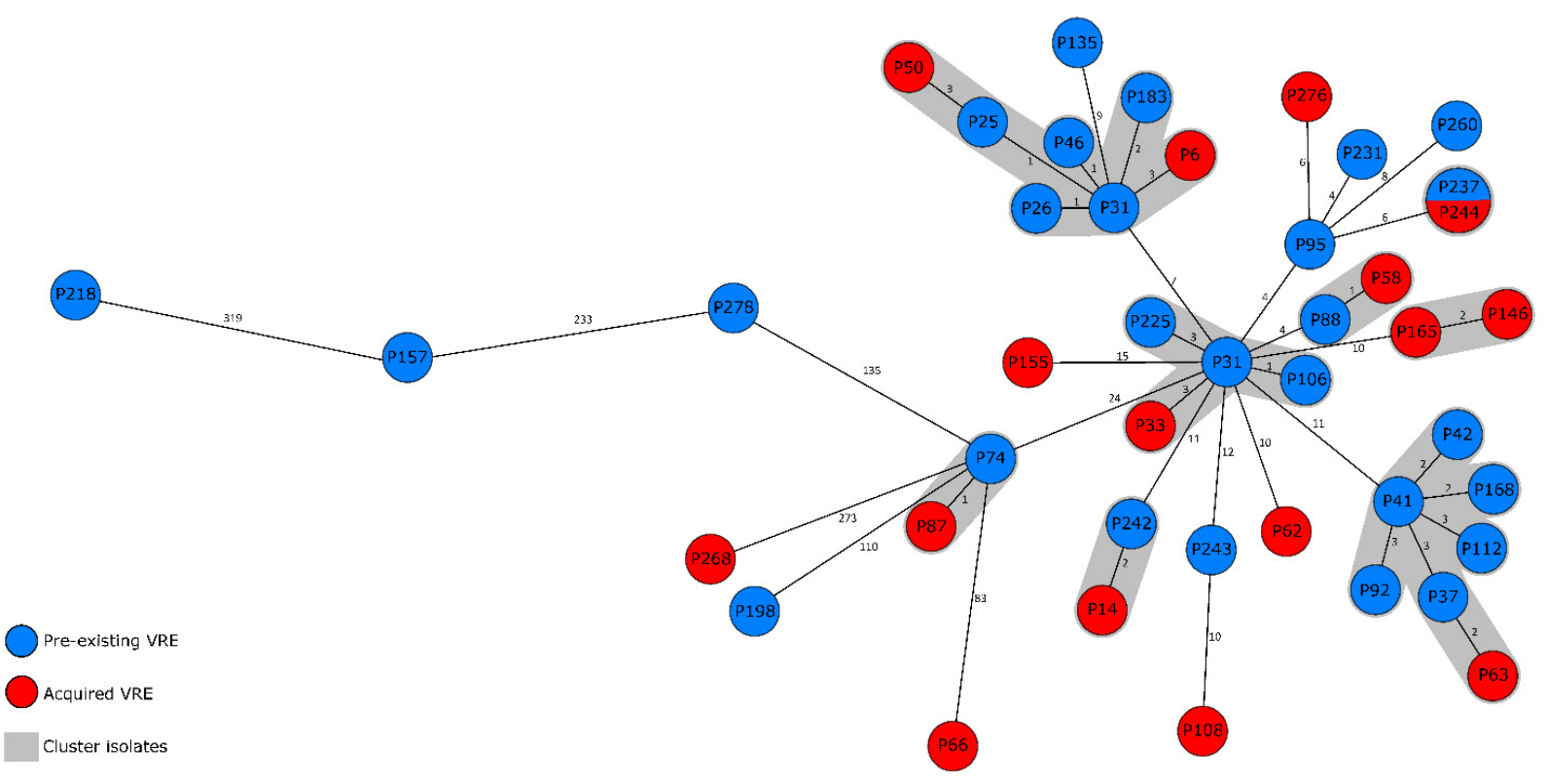

Figure 2. Minimum spanning tree of 43 preexisting $(n=27$, blue) and acquired $(n=16$, red) VRE strains based on 1423 target genes pairwise, ignoring missing values and numbered consecutively. Each circle represents one genotype. Connecting lines between genotypes indicate the number of allelic differences. Grey shadings illustrate close genetic relations ( $\leq 3$ alleles difference between directly connected genotypes). 


\section{Discussion}

VRE are an emerging issue to public health worldwide and were therefore classified as microorganisms of high-level priority by the World Health Organization in 2017 [19]. As hospital-acquired VRE are a result of interactions between host-associated factors, such as antibiotic selection and intra-hospital transmission $[20,21]$ we assessed (i) whether oral vancomycin facilitates the acquisition of VRE compared to metronidazole treatment and (ii) if there are pathogen-specific genetic differences favoring VRE acquisition in CDI patients.

In our study, oral vancomycin did not pose a predisposition for VRE acquisition compared to metronidazole. These results correspond with previous studies. Possible sources of bias in these studies are the analysis of VRE acquisition during CDI prophylaxis with vancomycin [22] and the gender distribution of a patient cohort with more than $90 \%$ of male individuals [12,23]. Nevertheless, available data surprisingly do not suggest any association between vancomycin administration and hospital-acquired VRE in CDI affected patients, whereby studies in non-CDI-patients have verified this association [23,24]. This might be due to the significantly lower prevalence of VRE in non-CDI patients, which was $<1 \%$ in our study period and connotes that CDI-patients are at special risk to acquire VRE.

Moreover, our cgMLST analysis of VRE strains revealed no specific relatedness or clearly distinguished pattern acquired from preexisting VRE isolates. This renders VRE genetic factors unlikely to favor VRE acquisition under CDI treatment. Hence, a more complex interplay of risk factors for this phenomenon needs to be considered, including transmissions via patient surroundings [25] and host gut microbiome changes due to additional antimicrobial treatments [20] facilitating VRE colonization. This context, e.g., the application of cephalosporines, allows VRE selection due to possible disturbance of the normal gut flora [26] and emphasizes the importance of antimicrobial stewardship (AMS) approaches. Our cgMLST analysis did additionally uncover close genetic relations and thereby the possibility of intra-hospital transmissions among isolates originating from patients with preexisting and acquired colonization, supporting the necessity of AMS teams working hand-in=hand with classical infection control specialists for preventing VRE hospital spread. Of note, we mainly determined vanB-positive strains comprising ST117, an observation that comes along with current trends in Germany [27] and raises the question whether VRE of this genetic composition do generally have a selective advantage in the hospital setting. Further comparative studies will be needed to address this aspect.

Our study has limitations. As we performed a retrospective observational study, this comes along with common constraints compared with randomized controlled studies. Moreover, we cannot assure that acquired VRE has no association with patient-patient or surface-patient transmission. Prior studies have shown that contact with a confirmed VRE patient is a major risk factor for VRE acquisition [28]. Therefore, we have to rely on the existing VRE control bundle to reduce transmission within the hospital as best as possible. Furthermore, detection of phenotypic vancomycin resistant isolates in the described matter is a limitation, since there was the necessity to select only a few isolated colonies [29]. Nevertheless, up till now, this describes the standard antimicrobial susceptibility testing, thereby offering the possibility of comparisons.

\section{Materials and Methods}

\subsection{Setting and Study Design}

The UHM is a 1427-bed tertiary care center with 57,132 patient admissions in 2019 [30]. In this setting, we conducted a 2-year retrospective cohort study from 2018 to 2020 . Patients with episodes of toxin positive CDI receiving metronidazole or oral vancomycin for CDI-specific treatment were included. VRE status at the beginning of CDI treatment was compiled in order to differentiate between patients with and without preexisting VRE colonization. Patients' characteristics, including demographic data (age, sex) and risk factors for VRE acquisition, such as underlying immunosuppressive diseases (e.g., malignancies, HIV-infection, liver transplantation) and treatments (e.g., long-term dialysis, systemic steroid therapy), were monitored. CDI cases were classified as severe if patients had 
to be admitted from an ambulatory setting, were transferred to an intensive care unit, underwent colorectal surgery, or died due to CDI infection [2]. CDI was assumed to be hospital-acquired if detected $48 \mathrm{~h}$ post-admission.

\subsection{Infection Control Measures}

In the case of VRE (colonization/infection) or toxin-positive CDI detection, extended hygiene measures were implemented, including contact isolation of patients in separate rooms and extra sanitary facilities. All staff members were advised to wear personal protective equipment, comprising gowns and gloves. Surface disinfection was performed at least once a day. In case of CDI, disinfection was performed with sporicidal disinfectants. Patient with VRE colonization were de-isolated if three anorectal swabs taken in consecutive weeks and without application of antibiotic therapy were negative for VRE. In the case of toxin-positive CDI, isolation was discontinued for patients free of CDI-associated symptoms for at least $48 \mathrm{~h}$.

\subsection{Diagnostic Procedure}

Unformed stool samples were analyzed in a two-step process for toxins producing C. difficile following the current ESCMID standards [4]. In brief, first glutamate dehydrogenase (GDH) was detected using the VIDAS ${ }^{\circledR}$ C. difficile GDH system (bioMérieux, Nürtingen, Germany). If GDH positive, C. difficile toxin was detected using the Xpert ${ }^{\circledR}$ C. difficile BT system (Cepheid ${ }^{\circledR}$, Krefeld, Germany) for confirmation.

\subsection{VRE Screening, Culture, and Antimicrobial Susceptibility Testing}

VRE screening was performed obtaining rectal swabs $\left(5 \mathrm{~cm} a b\right.$ ano) (Transwab ${ }^{\circledR}$ m40 compliant, mwe, Corsham, Wiltshire, UK), which were streaked to chromogenic selective agar (VRESelect ${ }^{\mathrm{TM}}$, Biorad, München, Germany) and incubated for $48 \mathrm{~h}$ at $36^{\circ} \mathrm{C}$. Suspected VRE colonies were verified on a species level using MALDI-TOF MS (Bruker Corporation, Bremen, Germany). Antibiotic susceptibility was evaluated in accordance with the current recommendations of the European Committee on Antimicrobial Susceptibility Testing (EUCAST) [31] using the VITEK ${ }^{\circledR} 2$ system and the minimal inhibition concentration (bioMérieux, Nürtingen, Germany). Confirmation of species identification and glycopeptide resistance $(\operatorname{van} A, \operatorname{van} B, \operatorname{van} C 1$, and $\operatorname{van} C 2 / 3)$ was done using the GenoType Enterococcus ${ }^{\circledR}$ line probe (Hain Lifescience, Nehren, Germany).

\subsection{Whole Genome Sequencing}

VRE isolates from CDI patients were collected for subsequent genetic comparison purposes, including those of patients with colonization prior and posterior to CDI treatment. To elucidate the genetic relationship, isolates were subjected to Whole Genome Sequencing (WGS) using either the Illumina MiSeq or the HiSeq platform (Illumina Inc., San Diego, CA, USA). After quality trimming, coding core genome regions were compared in a geneby-gene approach (core genome multilocus sequence typing, cgMLST) with the help of the SeqSphere + software version 7.0.1 (Ridom GmbH, Münster, Germany), using the published E. faecium cgMLST target scheme [32]. The clonal relationship of genotypes is displayed via a minimum spanning tree algorithm using the same software and is considered closely related if genotypes differ in three alleles or less. The MLST sequence types (STs) and underlying van-genes were extracted from the WGS data in silico.

\subsection{Statistical Analysis}

All data are expressed as absolute numbers or percentage. Categorical data were analyzed using Fischer's exact test. Statistical significance was assumed at $p<0.05$.

\subsection{Ethical Statement}

All strategies and investigations were performed in accordance with the national recommendations for surveillance of nosocomial infections and multidrug-resistant bacteria 
of the German legally-assigned institute for infection control and prevention (Robert-Koch Institute, Berlin, Germany).

\section{Conclusions}

Antibiotic treatment of CDI-affected patients can favor the acquisition of VRE. Therefore, compared to metronidazole, oral vancomycin does not increase the risk of VRE. No specific genetic features underlie the acquisition of VRE. Rather, this results from both antibiotic selection and pathogen transmission, strengthening the essential need of infection prevention bundle strategies consisting of antibiotic stewardship programs and infection control measures.

Author Contributions: Conceptualization, S.K.; formal analysis, N.C.J.H.; investigation, C.L.C.-M., N.C.J.H., N.J.F. and S.K.; data curation, S.K.; writing-original draft preparation, C.L.C.-M., N.C.J.H. and S.K.; writing — review and editing, C.L.C.-M., N.J.F. and S.K.; visualization, S.K.; supervision, S.K. All authors have read and agreed to the published version of the manuscript.

Funding: This research received no external funding.

Institutional Review Board Statement: Not applicable.

Informed Consent Statement: All strategies and investigations were performed in accordance with the national recommendations for surveillance of nosocomial infections and multidrug-resistant bacteria of the German legally-assigned institute for infection control and prevention (Robert-Koch Institute). Formal consent was therefore not required.

Data Availability Statement: Data is contained within the article.

Acknowledgments: We thank Ursula Keckevoet, Isabell Höfig, and Jessica Engberding for excellent technical laboratory assistance.

Conflicts of Interest: The authors declare no conflict of interest.

\section{References}

1. KISS Krankenhaus-Infektions-Surveillance-System-Modul CDAD-KISS Referenzdaten. Available online: https://www.nrzhygiene.de/fileadmin/nrz/module/cdad/202001_202012CDAD_Ref.pdf (accessed on 20 October 2021).

2. Gesetz zur Verhütung und Bekämpfung von Infektionskrankheiten beim Menschen (Infektionsschutzgesetz-IfSG). Available online: https:/ / www.gesetze-im-internet.de/ifsg/IfSG.pdf (accessed on 20 October 2021).

3. Johnson, S.; Lavergne, V.; Skinner, A.M.; Gonzales-Luna, A.J;; Garey, K.W.; Kelly, C.P.; Wilcox, M.H. Clinical Practice Guideline by the Infectious Diseases Society of America (IDSA) and Society for Healthcare Epidemiology of America (SHEA): 2021 Focused Update Guidelines on Management of Clostridioides difficile Infection in Adults. Clin. Infect. Dis. 2021, 73, e1029-e1044. [CrossRef]

4. Debast, S.; Bauer, M.; Kuijper, E. European Society of Clinical Microbiology and Infectious Diseases: Update of the Treatment Guidance Document for Clostridium difficile Infection. Clin. Microbiol. Infect. 2014, 20, 1-26. [CrossRef] [PubMed]

5. Drekonja, D.M.; Butler, M.; MacDonald, R.; Bliss, D.; Filice, G.A.; Rector, T.S.; Wilt, T.J. Comparative effectiveness of Clostridium difficile treatments: A systematic review. Ann. Intern. Med. 2011, 155, 839-847. [CrossRef] [PubMed]

6. Nelson, R.L.; Suda, K.J.; Evans, C.T. Antibiotic treatment for Clostridium difficile-associated diarrhoea in adults. Cochrane Database Syst. Rev. 2017, 2017, CD004610. [CrossRef]

7. Cho, J.M.; Pardi, D.S.; Khanna, S. Update on Treatment of Clostridioides difficile Infection. Mayo Clin. Proc. 2020, 95, 758-769. [CrossRef] [PubMed]

8. Gerding, D.N. Is there a relationship between vancomycin-resistant enterococcal infection and Clostridium difficile infection? Clin. Infect. Dis. 1997, 25 (Suppl. 2), S206-S210. [CrossRef]

9. Carmeli, Y.; Samore, M.H.; Huskins, C. The association between antecedent vancomycin treatment and hospital-acquired vancomycin-resistant enterococci: A meta-analysis. Arch. Intern. Med. 1999, 159, 2461-2468. [CrossRef] [PubMed]

10. Correa-Martinez, C.L.; Stollenwerk, V.B.; Kossow, A.; Schaumburg, F.; Mellmann, A.; Kampmeier, S. Risk Factors for Long-Term Vancomycin-Resistant Enterococci Persistence-A Prospective Longitudinal Study. Microorganisms 2019, 7, 400. [CrossRef]

11. DiNubile, M.J.; Chow, J.; Satishchandran, V.; Polis, A.; Motyl, M.R.; Abramson, M.A.; Teppler, H. Acquisition of Resistant Bowel Flora during a Double-Blind Randomized Clinical Trial of Ertapenem versus Piperacillin-Tazobactam Therapy for Intraabdominal Infections. Antimicrob. Agents Chemother. 2005, 49, 3217-3221. [CrossRef]

12. Stevens, V.W.; Khader, K.; Echevarria, K.; Nelson, R.E.; Zhang, Y.; Jones, M.; Timbrook, T.T.; Samore, M.H.; Rubin, M.A. Use of Oral Vancomycin for Clostridioides difficile Infection and the Risk of Vancomycin-Resistant Enterococci. Clin. Infect. Dis. 2019, 71, 645-651. [CrossRef] 
13. Prematunge, C.; MacDougall, C.; Johnstone, J.; Adomako, K.; Lam, F.; Robertson, J.; Garber, G. VRE and VSE Bacteremia Outcomes in the Era of Effective VRE Therapy: A Systematic Review and Meta-analysis. Infect. Control. Hosp. Epidemiol. 2015, 37, 26-35. [CrossRef]

14. DiazGranados, C.A.; Zimmer, S.M.; Mitchel, K.; Jernigan, J.A. Comparison of Mortality Associated with Vancomycin-Resistant and Vancomycin-Susceptible Enterococcal Bloodstream Infections: A Meta-analysis. Clin. Infect. Dis. 2005, 41, 327-333. [CrossRef]

15. Zacharioudakis, I.M.; Zervou, F.N.; Ziakas, P.; Rice, L.B.; Mylonakis, E. Vancomycin-Resistant Enterococci Colonization Among Dialysis Patients: A Meta-analysis of Prevalence, Risk Factors, and Significance. Am. J. Kidney Dis. 2015, 65, 88-97. [CrossRef] [PubMed]

16. Papadimitriou-Olivgeris, M.; Drougka, E.; Fligou, F.; Kolonitsiou, F.; Liakopoulos, A.; Dodou, V.; Anastassiou, E.D.; Petinaki, E.; Marangos, M.; Filos, K.S.; et al. Risk factors for enterococcal infection and colonization by vancomycin-resistant enterococci in critically ill patients. Infection 2014, 42, 1013-1022. [CrossRef]

17. Erb, S.; Frei, R.; Dangel, M.; Widmer, A.F. Multidrug-Resistant Organisms Detected More Than 48 Hours after Hospital Admission Are Not Necessarily Hospital-Acquired. Infect. Control. Hosp. Epidemiol. 2016, 38, 18-23. [CrossRef]

18. Suleyman, G.; Alangaden, G.; Bardossy, A.C. The Role of Environmental Contamination in the Transmission of Nosocomial Pathogens and Healthcare-Associated Infections. Curr. Infect. Dis. Rep. 2018, 20, 12. [CrossRef]

19. Tacconelli, E.; Carrara, E.; Savoldi, A.; Harbarth, S.; Mendelson, M.; Monnet, D.L.; Pulcini, C.; Kahlmeter, G.; Kluytmans, J.; Carmeli, Y.; et al. Discovery, research, and development of new antibiotics: The WHO priority list of antibiotic-resistant bacteria and tuberculosis. Lancet Infect. Dis. 2018, 18, 318-327. [CrossRef]

20. Monteserin, N.; Larson, E. Temporal trends and risk factors for healthcare-associated vancomycin-resistant enterococci in adults. J. Hosp. Infect. 2016, 94, 236-241. [CrossRef] [PubMed]

21. Ford, C.D.; Lopansri, B.K.; Gazdik, M.A.; Webb, B.; Snow, G.L.; Hoda, D.; Adams, B.; Petersen, F.B. Room contamination, patient colonization pressure, and the risk of vancomycin-resistant Enterococcus colonization on a unit dedicated to the treatment of hematologic malignancies and hematopoietic stem cell transplantation. Am. J. Infect. Control 2016, 44, 1110-1115. [CrossRef] [PubMed]

22. Babar, S.; El Kurdi, B.; El Iskandarani, M.; Haddad, I.; Imam, Z.; AlOmari, M.; Myers, J.; Moorman, J. Oral vancomycin prophylaxis for the prevention of Clostridium difficile infection: A systematic review and meta-analysis. Infect. Control Hosp. Epidemiol. 2020, 41, 1302-1309. [CrossRef] [PubMed]

23. Correa-Martínez, C.L.; Schuler, F.; Kampmeier, S. Sex differences in vancomycin-resistant enterococci bloodstream infections-A systematic review and meta-analysis. Biol. Sex Differ. 2021, 12, 36. [CrossRef] [PubMed]

24. Remschmidt, C.; Behnke, M.; Kola, A.; Diaz, L.A.P.; Rohde, A.M.; Gastmeier, P.; Schwab, F. The effect of antibiotic use on prevalence of nosocomial vancomycin-resistant enterococci-An ecologic study. Antimicrob. Resist. Infect. Control 2017, 6, 95. [CrossRef] [PubMed]

25. Correa-Martinez, C.L.; Tönnies, H.; Froböse, N.J.; Mellmann, A.; Kampmeier, S. Transmission of Vancomycin-Resistant Enterococci in the Hospital Setting: Uncovering the Patient-Environment Interplay. Microorganisms 2020, 8, 203. [CrossRef]

26. Faron, M.L.; Ledeboer, N.A.; Buchan, B.W. Resistance Mechanisms, Epidemiology, and Approaches to Screening for VancomycinResistant Enterococcus in the Health Care Setting. J. Clin. Microbiol. 2016, 54, 2436-2447. [CrossRef] [PubMed]

27. Eigenschaften, Häufigkeit und Verbreitung von Vancomycin-resistenten Enterokokken (VRE) in Deutschland. Update 2019/20. Available online: https://www.rki.de/DE/Content/Infekt/EpidBull/Archiv/2021/Ausgaben/27_21.pdf?_blob= publicationFile (accessed on 20 October 2021).

28. Flokas, M.E.; Karageorgos, S.A.; Detsis, M.; Alevizakos, M.; Mylonakis, E. Vancomycin-resistant enterococci colonisation, risk factors and risk for infection among hospitalised paediatric patients: A systematic review and meta-analysis. Int. J. Antimicrob. Agents 2017, 49, 565-572. [CrossRef]

29. Brukner, I.; Oughton, M. A Fundamental Change in Antibiotic Susceptibility Testing Would Better Prevent Therapeutic Failure: From Individual to Population-Based Analysis. Front. Microbiol. 2020, 11, 1820. [CrossRef] [PubMed]

30. Referenzbericht Universitätsklinikum Münster 2019. Available online: https://www.ukm.de/fileadmin/ukminternet/daten/ zentralauftritt/patienten-besucher/Qualtitaet_Hygiene/ukm-qualitaetsbericht-2019.pdf (accessed on 20 October 2021).

31. Breakpoint Tables for Interpretation of MICs and Zone Diameters Version 11.0. Available online: https://www.eucast.org/ fileadmin/src/media/PDFs/EUCAST_files/Breakpoint_tables/v_11.0_Breakpoint_Tables.pdf (accessed on 20 October 2021).

32. de Been, M.; Pinholt, M.; Top, J.; Bletz, S.; Mellmann, A.; van Schaik, W.; Brouwer, E.; Rogers, M.; Kraat, Y.; Bonten, M.; et al. Core Genome Multilocus Sequence Typing Scheme for High-Resolution Typing of Enterococcus faecium. J. Clin. Microbiol. 2015, 53, 3788-3797. [CrossRef] 